\title{
Laser Generated Ultrasound Sources Using Polymer Nanocomposites for High Frequency Metrology
}

\author{
Srinath Rajagopal ${ }^{1,2}$, Toby Sainsbury ${ }^{3}$, Bradley E. Treeby ${ }^{1}$ and Ben T. Cox ${ }^{1}$ \\ ${ }^{1}$ Department of Medical Physics and Biomedical Engineering, University College London, London, U.K. \\ ${ }^{2}$ Ultrasound and Underwater Acoustics, National Physical Laboratory, Teddington, U.K. E: srinath.rajagopal@npl.co.uk \\ ${ }^{3}$ Catalysis Center and Division of Physical Sciences and Engineering, KUAST, Thuwal, Kingdom of Saudi Arabia.
}

\begin{abstract}
Accurate characterization of ultrasound fields generated by diagnostic and therapeutic transducers is critical for patient safety. This requires hydrophones calibrated to a traceable standard and currently the upper calibration frequency range available to the user community is limited to a frequency of $40 \mathrm{MHz}$. However, the increasing use of high frequencies for both imaging and therapy necessitates calibrations to frequencies well beyond this range. For this to be possible, a source of high amplitude, broadband, quasi-planar and stable ultrasound fields is required. This is difficult to achieve using conventional piezoelectric sources, but laser generated ultrasound is a promising technique in this regard. In this study, various polymer-carbon nanotube nanocomposites (PNC) were fabricated and tested for their suitability for such an application by varying the polymer type, carbon nanotubes weight content in the polymer, and PNC thickness. A broadband hydrophone was used to measure the peak pressure and bandwidth of the laser generated ultrasound pulse. Peak-positive pressures of up to $8 \mathrm{MPa}$ and $-6 \mathrm{~dB}$ bandwidths of up to $40 \mathrm{MHz}$ were recorded. There is a nonlinear dependence of the peak pressure on the laser fluence and the bandwidth scales inversely proportionally to the peak pressure. The high-pressure plane waves generated from this preliminary investigation has demonstrated that laser generated ultrasound sources are a promising technique for high frequency calibration of hydrophones.
\end{abstract}

\section{INTRODUCTION}

Ultrasound is a successful biomedical imaging modality and its ubiquitous use in modern healthcare is largely because it is real-time, portable and relatively cheap. Measurement of ultrasound fields generated by diagnostic and therapeutic medical ultrasound equipment is crucial to ensure patient safety [1][2]. In addition, measurement is also needed for performance characterization by equipment manufacturers to demonstrate compliance for regulatory purposes and in research and development. Traditionally, hydrophones used to characterize the ultrasound fields with a known frequency response up to $40 \mathrm{MHz}$ was deemed sufficient [3]. However, recent advancements in high frequency applications of ultrasound in diagnosis [4] and the use of high intensity ultrasound for therapy [5] demands hydrophone responses be known to frequencies as high as $100 \mathrm{MHz}$ for accurate characterization of the field.

The calibration of hydrophones is currently undertaken in ultrasound fields such as nonlinearly-steepened tone-burst wave, broadband pulses, and swept frequency chirps [6]. It is required that the ultrasound beam is spatially broad such that it minimizes the error due to spatial averaging by the finite sized sensing aperture of the hydrophone and is also of sufficiently high pressure to overcome the signal-to-noise (SNR) of the displacement or velocity sensing interferometer [6]. At frequencies beyond $50 \mathrm{MHz}$ it is not trivial to generate an acoustic field that satisfies both SNR and spatial broadness using piezo transducer technology. However, laser generated ultrasound is a promising technique in this regard. It is possible to generate a broadband and high amplitude ultrasound field using pulsed laser excitation of a light-absorbing material via thermo-elastic expansion [7].

The aim of the present research was to investigate the requirements of carbon nanotubes and polymer based nanocomposites excited using a pulsed laser as a potential source of broadband and high amplitude planar ultrasound pulses for use in calibrating hydrophones.

\section{MATERIALS AND METHODS}

\section{A. Carbon-Polymer Nanocomposites}

The multi-walled carbon nanotubes (CNTs), polymer and catalyst (curing agent) were all combined by their mass fractions. The CNTs were dispersed in the polymer using shear mixing at $3500 \mathrm{rpm}$ for $2 \mathrm{~min}$ (DAC $150.1 \mathrm{FV}-\mathrm{K}$, SpeedMixer ${ }^{\mathrm{TM}}$, High Wycombe, U.K.). The catalyst was then added and shear mixed again at $3500 \mathrm{rpm}$ for $2 \mathrm{~min}$. Functionalized [8][9] CNTs were used to prevent their agglomeration in polymer when shear mixing. The CNTpolymer mixture was used to coat a laboratory-grade glass slide using a blade film applicator (Sheen Instruments, West Molesey, U.K). In this technique, a height adjustable knife edged metal frame attached to a micrometer controls the gap clearance relative to a flat surface e.g., a glass slide. A thin film is produced when the excess mixture smeared on the glass slide is removed by sliding the knife edged metal frame over the length of the glass slide. A polymer nanocomposite (PNC) source was formed after oven curing the thin film (Table 1).

The initial photoacoustic pressure distribution, $p_{0}(x)$ developed within the PNC source in the absence of optical scatter following optical absorption is given by Beer-Lambert relation:

$$
p_{0}(x)=\Phi \cdot \mu_{\mathrm{a}} \cdot \Gamma \cdot \exp \left(\mu_{\mathrm{a}} \cdot x\right)
$$


TABLE I. POLYMER TYPES USED IN THE PREPARATION OF PNC.

\begin{tabular}{llll}
\hline Polymer type & Product & $\begin{array}{l}\text { Mix ratio } \\
\text { (base:catalyst) }\end{array}$ & Curing conditions \\
\hline Epoxy & Araldite LY-564 & $2.86: 1$ & $\begin{array}{l}\text { Oven cured for 12 } \\
\text { hours at 70 C }\end{array}$ \\
\hline $\begin{array}{l}\text { Polydimethylsiloxane } \\
\text { (PDMS) }\end{array}$ & SYLGARD® 184 & $5: 1$ & $\begin{array}{l}\text { Oven cured for 35 } \\
\text { mins at 100 C }\end{array}$ \\
\hline $\begin{array}{l}\text { Polyurethane } \\
\text { (PU) }\end{array}$ & Polycraft & $1: 1$ & $\begin{array}{l}\text { Oven cured for 12 } \\
\text { hours at 40 C }\end{array}$ \\
\hline
\end{tabular}

where, $\Gamma=\beta c_{0}^{2} / C_{\mathrm{p}}$ is the dimensionless thermodynamic efficiency coefficient known as the Grüneisen parameter of the PNC source, $\mu_{\mathrm{a}}$ is the optical absorption coefficient in $\mathrm{m}^{-1}$ and $\Phi$ is the laser fluence in $\mathrm{J}^{-\mathrm{m}^{-2}}$. Here, $\beta$ is the volume thermal expansion coefficient in $\mathrm{K}^{-1}, c_{0}$ is the sound speed in $\mathrm{m}^{-1} \mathrm{~s}^{-1}$ and $C_{\mathrm{p}}$ is the isobaric heat capacity in $\mathrm{J}_{\mathrm{kg}}^{-1} \cdot \mathrm{K}^{-1}$. The $\beta$ of polymers can vary by a factor of 10 across different types, significantly affecting the amplitude of $p_{0}(x)$ compared to other parameters of $\Gamma$. A total of $27 \mathrm{PNC}$ sources were prepared by varying the polymer type (epoxy, polyurethane (PU) and polydimethylsiloxane (PDMS)), weight fraction (wt\%) of CNTs in the polymer $(1.25,2.5$ and $3.5 \mathrm{wt} \%)$ and the composite thickness $(18-30 \mu \mathrm{m}, 40-60 \mu \mathrm{m}$ or $50-70 \mu \mathrm{m})$.

\section{B. Experimental Setup}

The test setup used to measure the LGUS responses from PNC sources is shown in Fig. 1. A pulsed laser (Nano 120-S, Litron Ltd, U.K.) operating at $1064 \mathrm{~nm}$ with a full-width halfmaximum of $\sim 4 \mathrm{~ns}$ and a peak energy of $120 \mathrm{~mJ}$ per pulse was used for the study. The expanded beam from the laser was homogenized using a 1500 grit ground glass optical diffuser (ODI). The homogenized beam was weakly converged using a plano-convex lens of $100 \mathrm{~mm}$ focal length to minimize the losses due to scatter caused by the ODI before illuminating the beam through the clear polymethyl methacrylate (PMMA) wall of the water tank. A membrane hydrophone (UT1602, Precision Acoustics Ltd, U.K) with a nominal element diameter

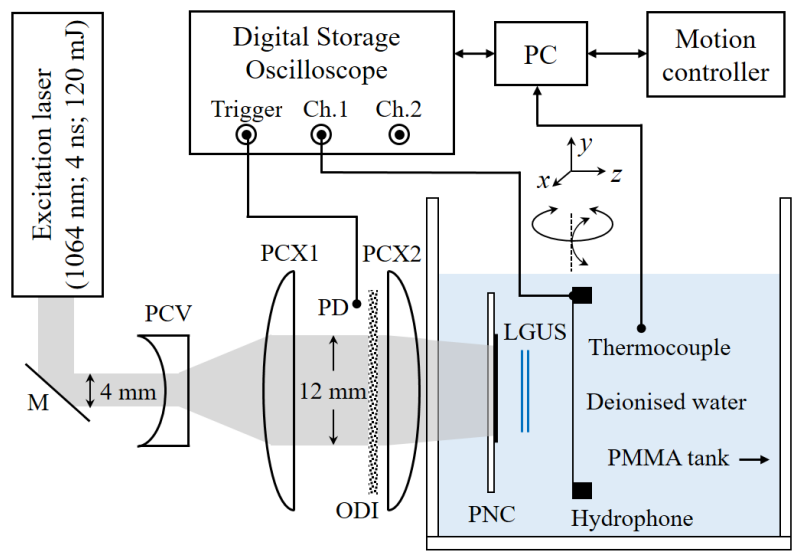

Fig. 1. Test setup. Mirror (M); plano-concave lens (PCV); plano-convex lens (PCX1 and PCX2); photodetector (PD); optical diffuser (ODI); CNT-polymer nanocomposite (PNC) source; laser generated ultrasound (LGUS); Polymethyl methacrylate (PMMA). of $0.2 \mathrm{~mm}$ was used to measure the LGUS response. The electrical response of the LGUS pulse acquired from the hydrophone was converted to a pressure pulse using the complex hydrophone sensitivity [10]. The hydrophone's calibration was traceable to primary standard from 1 to 60 $\mathrm{MHz}$ [11] and was also predicted from 1 to $110 \mathrm{MHz}$ using a 1D analytical model previously developed at National Physical Laboratory [12]. An agreement between the measured and modelled response was obtained by optimizing the model input parameters. A 5-axis gantry with rotation, tilt and three motorized linear axes (LNR50S/M, Thorlabs) was used for field scanning of the LGUS from the PNC sources using ultrasound measurement software (UMS2, Precision Acoustics Ltd, Dorchester, UK). A photodetector (PD) provided a trigger to the digital storage oscilloscope (TDS7254, Tektronix, Beaverton, USA). A thermocouple placed inside the PMMA tank was used to record the water temperature. A measurement record consisted of acquiring 32 LGUS pulses sequentially and correcting each pulse for fluctuations in the laser energy using the PD signal acquired simultaneously. The corrected LGUS pulses were averaged and stored for later analysis. All measurements were undertaken at a distance of $\sim 7.4 \mathrm{~mm}$ from the PNC source.

\section{Laser Fluence}

A calibrated pyroelectric energy sensor (ES220C, Thorlabs, U.K.) of $20 \mathrm{~mm}$ active diameter was used to measure the peak energy at the test site for a range of attenuator settings on the laser with no water in the PMMA tank. The beam-area was indirectly determined by scanning the LGUS field from an epoxy based PNC source using the hydrophone over an area of $1.5 \times 1.5 \mathrm{~cm}$ at $0.25 \mathrm{~mm}$ step size at an axial distance of $7.4 \mathrm{~mm}$. The raster scan was undertaken at $30 \%$ of the maximum energy level. The beam-area $(-20 \mathrm{~dB})$ was estimated such that it included all pixels of the raster scan that were greater than or equal to $10 \%$ (or $-20 \mathrm{~dB}$ ) of the peak value in the image. The estimated beam area $\left(0.81 \mathrm{~cm}^{2}\right)$ was used to calculate the fluence at the measured energy levels.

\section{RESULTS AND DISCUSSION}

The LGUS response from the PNC sources was obtained by deconvolving the measured hydrophone response and how they vary with polymer type, CNT wt $\%$, film thickness, and fluence were examined.

\section{A. Effect of Polymer Type}

The effect of the polymer's volume thermal expansion coefficient, $\beta$, on the LGUS pulse can be seen in Fig. 2. The epoxy, PU and PDMS based PNC sources were loaded with $3.5 \mathrm{wt} \% \mathrm{CNT}$, with a nominal thickness of $55 \mu \mathrm{m}$ and were tested at an applied fluence of $10 \mathrm{~mJ} . \mathrm{cm}-2$. The $\beta$ of the PNC sources tested within this study was not measured but the known range for various types of pure epoxy, PU and PDMS are in the range $30-70,60-120$ and $310-340 \times 10^{-6} \mathrm{~K}^{-1}$ respectively, which correlates with the measured peak positive pressures of 1.6, 2.7 and 5.1 MPa respectively. 


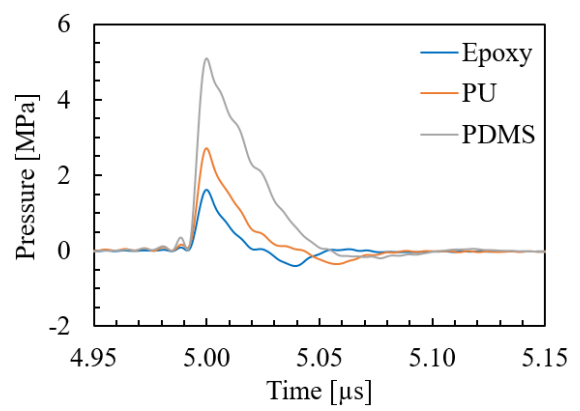

Fig. 2. Time-series showing the effect of polymer type on the laser generated ultrasound pulse. Epoxy, polyurethane (PU) and polydimethylsiloxane (PDMS) polymer nanocomposites with $3.5 \mathrm{wt} \%$ CNT, thickness of $\sim 55 \mu \mathrm{m}$ and an applied fluence of $10 \mathrm{~mJ} . \mathrm{cm}^{-2}$.

\section{B. Effect of CNT Weight Percent}

The measured optical absorption coefficient, $\mu_{\mathrm{a}}$ of the PNC sources of average thickness of $18 \pm 2 \mu \mathrm{m}$ with $1.25,2.5$ and $3.5 \mathrm{wt} \% \mathrm{CNT}$ in PDMS was 68, 167 and $245 \times 10^{3} \mathrm{~m}^{-1}$ respectively. According to (1), increasing the $\mu_{\mathrm{a}}$ of the PNC source should result in increased LGUS response. This is demonstrated in Fig. 3 for the $18 \mu \mathrm{m}$ PDMS based PNC source for an applied fluence of $20 \mathrm{~mJ} . \mathrm{cm}^{-2}$. The peak positive pressure for 1.25, 2.5 and $3.5 \mathrm{wt} \%$ CNT in PDMS was 4.6, 6.1 and 6.2 $\mathrm{MPa}$ respectively. It is seen that increasing the CNT weight content from 2.5 to $3.5 \mathrm{wt} \%$ in PDMS resulted in only a negligible increase in pressure. For efficient generation of LGUS it is critical that the acoustic propagation time across the optical absorption depth $\left(1 / \mu_{\mathrm{a}}\right)$ should be much greater than the heating time of the PNC medium, i.e., the duration of the laser pulse and the time for the absorbed optical energy to thermalize. This condition ensures that the heating of the medium is completed under isochoric conditions allowing maximum build-up of stress. Assuming the known speed of sound for example of pure PDMS $\left(1050 \mathrm{~m} \cdot \mathrm{s}^{-1}\right)$, the stress relaxation time $\left(1 / c_{0} . \mu_{\mathrm{a}}\right)$ for $1.25,2.5$ and $3.5 \mathrm{wt} \% \mathrm{CNT}$ in PDMS works out to be 14, 5.7 and 3.9 ns respectively. Clearly, the stress confinement criteria cannot be met for the case of 3.5 wt\% CNT since the heating time is longer than stress confinement time i.e., the medium starts to relax before heating of the medium is completed.

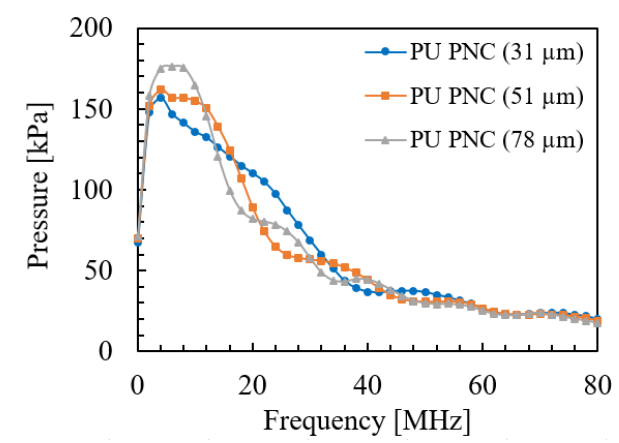

Fig. 4. Amplitude spectra from laser generated ultrasound pulses of polyurethane (PU) based polymer nanocomposite (PNC) sources of thicknesses of $\sim 31,51$ and $78 \mu \mathrm{m}$ with $1.25 \mathrm{wt} \% \mathrm{CNT}$ and for an applied fluence of $20 \mathrm{~mJ} . \mathrm{cm}^{-2}$.

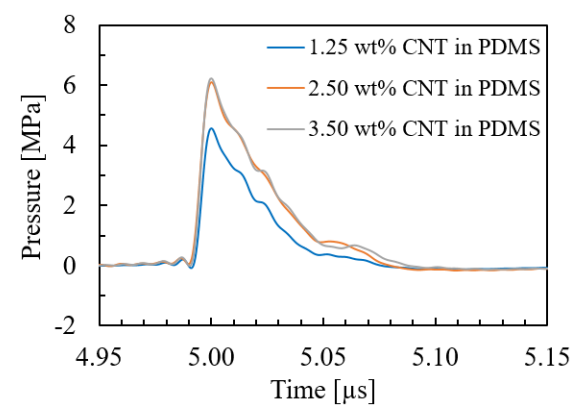

Fig. 3. Time-series showing the effect of $1.25,2.5$ and $3.5 \mathrm{wt} \%$ CNT in polydimethylsiloxane (PDMS) of $\sim 18 \mu \mathrm{m}$ thickness for an applied fluence of $20 \mathrm{~mJ} . \mathrm{cm}^{-2}$.

\section{Effect of PNC Source Thickness}

For a PNC medium with known optical absorption coefficient, $\mu_{\mathrm{a}}$ the thickness of the PNC source should be ideally equal to the absorption depth, $1 / \mu_{\mathrm{a}}$. Increasing the source thickness beyond $1 / \mu_{\mathrm{a}}$ will not contribute towards stress confinement but the additional PNC medium will attenuate the propagating acoustic wave through it. This is shown using the amplitude spectra in Fig. 4. of LGUS pulses for PU based PNC sources of thicknesses 31,51 and $78 \mu \mathrm{m}$ with $1.25 \mathrm{wt} \% \mathrm{CNT}$ and for an applied fluence of $20 \mathrm{~mJ} . \mathrm{cm}-2$. The calculated bandwidths for source thicknesses of 31,51 and $78 \mu \mathrm{m}$ were 28, 21 and $18 \mathrm{MHz}$ respectively. The loss of bandwidth with increasing thickness is a clear demonstration of acoustic attenuation within the PNC material.

\section{Effect of Laser Fluence}

Increasing the laser energy density $\left(\Phi . \mu_{\mathrm{a}}\right)$, in a PNC by increasing the laser fluence, $\Phi$, according to (1), will increase the amplitude of the initial pressure distribution, $p_{0}(x)$. The effect of laser fluence for the case of epoxy PNC with 1.25 wt $\% \mathrm{CNT}$ and $27 \mu \mathrm{m}$ thick are shown in the normalized amplitude spectra (Fig. 5) as an example for applied fluence of $10,20,30$ and $40 \mathrm{~mJ} . \mathrm{cm}^{-2}$. The increase in peak positive pressure with fluence was 1.2, 2.0, 2.7 and $3.2 \mathrm{MPa}$, which is nonlinear and the corresponding bandwidths were 36, 30, 26 and $21 \mathrm{MHz}$ respectively. The increase in pressure amplitude with fluence was accompanied with a decrease in bandwidth

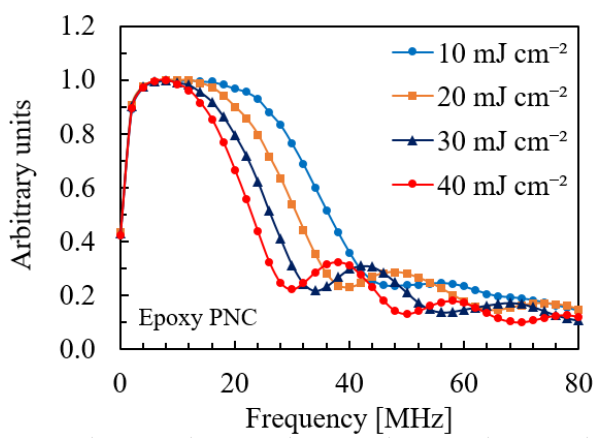

Fig. 5. Normalized amplitude spectra from laser generated ultrasound pulses of epoxy based polymer nanocomposite source of thickness $\sim 27$ $\mu \mathrm{m}$ with $1.25 \mathrm{wt} \% \mathrm{CNT}$. 
due to broadening of the time-series pressure pulse. The nonlinear response from PNC sources made of allotropes of carbon to the applied fluence has also been previously reported [13][14].

\section{E. Performance Comparison of PNC Sources}

In the preceding sections the effect of polymer, CNT wt $\%$, PNC thickness and fluence were discussed separately. The CNT wt $\%$ in a polymer determines the optical absorption depth. Therefore, in order to maximize the stress confinement, the laser pulse duration must be shorter than the transit time of the acoustic wave across the absorption depth, i.e., CNT wt\% and laser pulse duration are inversely proportional. Also, the thickness of the PNC source should ideally be equal to the optical absorption depth since the PNC medium beyond the optical absorption depth does not contribute to the stress confinement but the additional PNC medium will attenuate the propagating acoustic wave through it. As the acoustic loss increases rapidly with frequency in polymeric materials compared to pure water, the acoustic pressure and bandwidth both decrease with increasing thickness. The other two parameters i.e., the volume thermal expansion coefficient and fluence, affect the LGUS response significantly compared to CNT wt\% and PNC thicknesses considered in this study. The peak-positive pressure of the time-series LGUS pulse and its bandwidth for epoxy, PU and PDMS based PNC sources of nominally identical thicknesses and with CNT wt\% of 1.25 are shown in Fig. 6. The inverse relationship of pressure and bandwidth was a characteristic behavior observed across all 27 samples. The physical reasons for this are currently being investigated but nonlinear propagation is certainly one contributing factor.

\section{CONCLUSIOSNS}

Laser generated ultrasound pulses from CNT-polymer nanocomposite sources backed on a laboratory-grade glass slide were examined as a function of polymer type, CNT weight content in the polymer, source thickness and laser fluence. The amplitude of the LGUS was found to be directly proportional to polymer's volume thermal expansion coefficient. For a given duration of the laser pulse, increasing the CNT content did not increase the LGUS response since the acoustic transit time across the optical absorption depth is shorter than the laser pulse duration, limiting the stress confinement due to medium relaxation. Similarly, the PNC source thickness should ideally be equal to optical absorption depth to minimize acoustic attenuation and loss of bandwidth. The peak pressure was found to be nonlinearly dependent on the laser fluence and the bandwidth scaled inversely proportionally to the peak pressure.

\section{ACKNOWLEDGMENT}

The UK Department for Business, Energy \& Industrial Strategy's funding of the National Measurement System is gratefully acknowledged. S.R. thanks National Physical Laboratory (NPL) for supporting his PhD with University College London and Composites, Adhesives and Polymeric Materials Group at NPL for providing access to laboratory facilities to undertake the nanocomposite work.

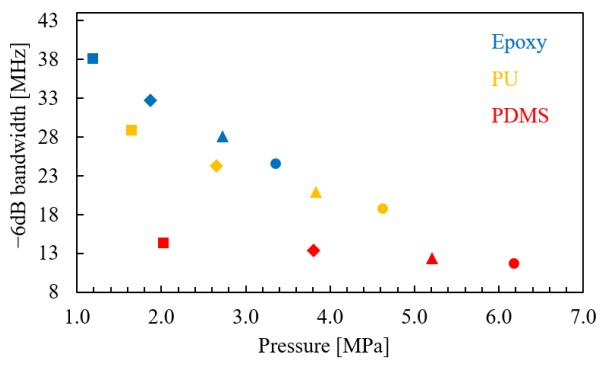

- $27 \mu \mathrm{m} ; 10 \mathrm{~mJ} \mathrm{~cm}{ }^{-2} \backsim 29 \mu \mathrm{m} ; 10 \mathrm{~mJ} \mathrm{~cm}{ }^{-2} \amalg 32 \mu \mathrm{m} ; 10 \mathrm{~mJ} \mathrm{~cm}^{-2}$ - $27 \mu \mathrm{m} ; 20 \mathrm{~mJ} \mathrm{~cm}^{-2} \diamond 29 \mu \mathrm{m} ; 20 \mathrm{~mJ} \mathrm{~cm}^{-2} \diamond 32 \mu \mathrm{m} ; 20 \mathrm{~mJ} \mathrm{~cm}^{-2}$ $\triangle 27 \mu \mathrm{m} ; 30 \mathrm{~mJ} \mathrm{~cm}^{-2} \Delta 29 \mu \mathrm{m} ; 30 \mathrm{~mJ} \mathrm{~cm}^{-2} \wedge 32 \mu \mathrm{m} ; 30 \mathrm{~mJ} \mathrm{~cm}^{-2}$ - $27 \mu \mathrm{m} ; 40 \mathrm{~mJ} \mathrm{~cm}^{-2} \bullet 29 \mu \mathrm{m} ; 40 \mathrm{~mJ} \mathrm{~cm}{ }^{-2} \bullet 32 \mu \mathrm{m} ; 40 \mathrm{~mJ} \mathrm{~cm}^{-2}$

Fig. 6. Scatter plot of bandwidth against pressure obtained from epoxy, polyurethane (PU) and polydimethylsiloxane (PDMS) polymer nanocomposite (PNC) sources with $1.25 \mathrm{wt} \% \mathrm{CNT}$ of nominally identical thicknesses for applied fluences of 10, 20,30 and $40 \mathrm{~mJ} . \mathrm{cm}^{-2}$ represented by square, diamond, triangle and circle respectively.

\section{REFERENCES}

[1] IEC, 60601-2-37:2007+AMD1 Medical electrical equipment - Part 2-37: Particular requirements for the basic safety and essential performance of ultrasonic medicaldiagnostic and monitoring equipment, 2015.

[2] IEC, 60601-2-62 Medical electrical equipment - Part 2-62: Particular requirements for the basic safety and essential performance of high intensity therapeutic ultrasound (HITU) equipment, 2013.

[3] [3] IEC, 62127 Ultrasonics - Hydrophones - Part 1: Measurement and characterisation of medical ultrasonic fields up to $40 \mathrm{MHz}, 2013$.

[4] K. K. Shung, "High Frequency Ultrasonic Imaging", Journal of Medical Ultrasound, vol. 17, no. 1, pp. 25-30, 2009.

[5] D. L. Miller et al., "Overview of therapeutic ultrasound applications and safety considerations.,” J. Ultrasound Med., 31 (4), pp. 623-34, 2012.

[6] IEC, 62127 Ultrasonics - Hydrophones - Part 2: Calibration for ultrasonic fields up to $40 \mathrm{MHz}, 2013$.

[7] S.-L. Chen, "Review of Laser-Generated Ultrasound Transmitters and Their Applications to All-Optical Ultrasound Transducers and Imaging," Appl. Sci., vol. 7, no. 1, p. 25, 2016.

[8] P.-C. Ma, N. A. Siddiqui, G. Marom, and J.-K. Kim, "Dispersion and functionalization of carbon nanotubes for polymer-based nanocomposites: A review," Compos. Part A Appl. Sci. Manuf., vol. 41, no. 10 , pp. $1345-1367,2010$.

[9] R. J. Zaldivar, J. P. Nokes, P. M. Adams, K. Hammoud, and H. I. Kim, "Surface functionalization without lattice degradation of highly crystalline nanoscaled carbon materials using a carbon monoxide atmospheric plasma treatment," Carbon, 50 (8), pp. 2966-2975, 2012.

[10] A. Hurrell and S. Rajagopal, "The practicalities of obtaining and using hydrophone calibration data to derive pressure waveforms," IEEE Trans. Ultrason. Ferroelectr. Freq. Control, vol. 64, no. 1, pp. 126-140, 2016.

[11] R. C. Preston, S. P. Robinson, B. Zeqiri, T. J. Esward, P. N. Gélat, and N. D. Lee, "Primary calibration of membrane hydrophones in the frequency range $0.5 \mathrm{MHz}$ to $60 \mathrm{MHz}$," Metrologia, vol. 36, no. 4, pp. 331-343, 2003.

[12] P. N. Gélat, R. C. Preston, and A. Hurrell, "A theoretical model describing the transfer characteristics of a membrane hydrophone and validation," Ultrasonics, vol. 43, no. 5, pp. 331-341, 2005.

[13] H. W. Baac et al., "Carbon-nanotube optoacoustic lens for focused ultrasound generation and high-precision targeted therapy.," Sci. Rep., vol. 2, p. 989, 2012.

[14] W. Y. Chang, W. Huang, J. Kim, S. Li, and X. Jiang, "Candle soot nanoparticles-polydimethylsiloxane composites for laser ultrasound transducers," Appl. Phys. Lett., vol. 107, no. 16, pp. 1-5, 2015. 